\title{
Atividade física relacionada ao estresse no trabalho dos profissionais da área da saúde mental
}

\author{
Physical activity related to stress among \\ mental health professionals
}

\author{
Bárbara Penha Verani, Melissa de Carvalho Souza', Silvia Rosane Parcias, Thiago Sousa Matias, \\ Adriana Coutinho de Azevedo Guimarães ${ }^{1}$ \\ 'Universidade do Estado de Santa Catarina (UDESC), Florianópolis, SC, Brasil.
}

Recebido em: junho 2015 / Aceito em: julho 2015

mecarvalho.s@gmail.com

\begin{abstract}
RESUMO
Objetivo: investigar a atividade física relacionada ao estresse no trabalho, dos profissionais que atendem pacientes com transtornos mentais e dependentes químicos. Método: para uma amostra não probabilística de 170 profissionais, utilizou-se questionário autoaplicável. A estatística foi descritiva e inferencial. Resultados e Considerações Finais: status de peso, nível de formação e estrato econômico obtiveram diferenças entre os sexos $(p<0,001 ; p=0,011 ; p=0,006)$. Houve correlação positiva entre caminhada e demanda controle e negativa com atividade física vigorosa. Não houve diferença entre os sexos quanto ao estresse. Atividade física parece não se relacionar com o estresse nesse momento, podendo haver a influência de variáveis não estudadas.
\end{abstract}

Palavras-chave: Atividade Física; Estresse Ocupacional; Saúde Mental.

\section{ABSTRACT}

Objective: investigate the physical activity related to stress at work of professionals who assist patients with mental disorders and drug addicts. Method: we used a self-administered questionnaire for a non-probabilistic sample with 170 professionals. Descriptive and inferential statistic was applied. Results and Closing Remarks: weight status, level of education and economic strata had differences between genders $(p$ $<0.001 ; p=0.011 ; p=0.006$, respectively). There was a positive correlation between walking and demand control and negative correlation with vigorous physical activity. There was no difference between genders as to stress. Physical activity appears to be not related to stress at this time, which may lead to influence of other variables not studied.

Keywords: Physical Activity; Occupational Stress; Mental Health.

\section{INTRODUÇÃO}

Atualmente, o trabalho parece ser um importante fator gerador de estresse, pois tornou-se uma importante fonte de preocupação e é reconhecido como um dos riscos mais sérios ao bem estar psicossocial do indivíduo'. Trabalhar na área da saúde tem algumas peculiaridades que colocam um fardo pesado de demandas físicas, cognitivas e emocionais sobre os profissionais desta área. ${ }^{2}$

O ambiente do centro hospitalar é considerado dinâmico, estimulante e heterogêneo, mas abrangendo simultaneamente, atividades insalubres, penosas e difíceis para todos os profissionais de saúde, gerando o estresse ocupacional que, frequentemente, está ligado à organização do trabalho, como carga de trabalho excessiva, más condições de trabalho, falta de treinamento e orientação, relação abusiva entre supervisores e subordinados, falta de controle sobre a tarefa e ciclos trabalho-descanso incoerentes com os limites biológicos, podendo dessa forma, estarem suscetíveis ao desenvolvimento do estresse. ${ }^{1}$

$\mathrm{O}$ estresse é considerado como qualquer estímulo que demande do ambiente externo ou interno e que taxe ou exceda as fontes de adaptação de um indivíduo ou sistema social. ${ }^{3}$ Devido a essa alta carga de estresse, imposta aos profissionais da saúde mental, há 
evidências que a atividade física cumpre um dos papéis mais importantes, que é a restauração da saúde, dos efeitos nocivos, que a rotina de trabalho estressante proporciona. ${ }^{4}$

$\mathrm{Na}$ busca por alternativas que valorizem a qualidade de vida dos trabalhadores, a atividade física é uma das opções que vem sendo apontada como favorecedora de melhorias e redução do estresse. Associada à hábitos de vida saudáveis pode ser importante fator na promoção da saúde, além de atuar na manutenção da condição de saúde no trabalho. ${ }^{5}$

Tendo em vista, de como se encontra a situação laboral dos profissionais da saúde mental, atribui-se à prática regular da atividade física, um meio para amenizar e resolver a problemática. $O$ objetivo do estudo foi investigar a prática de atividade física relacionada ao estresse no trabalho, dos profissionais que atendem pacientes com transtornos mentais e dependentes químicos.

\section{MÉTODO}

O estudo foi aprovado no Comitê de Ética em Pesquisa com Seres Humanos (CEPSH), da Universidade do Estado de Santa Catarina (UDESC), em Florianópolis, protocolo número 423.085. Caracterizou-se como quantitativo descritivo, com delineamento transversal. A amostra não probabilística por conveniência foi constituída por 170 profissionais de ambos os sexos, com média de idade de $36,5 \pm 9,96$ anos, da área da saúde mental. A coleta de dados ocorreu em dois dos maiores centros hospitalares de saúde mental, em número de pacientes e infraestrutura de São José - Santa Catarina.

Mediante a autorização das instituições envolvidas e após assinatura do termo de consentimento livre e esclarecido, por parte dos funcionários, o questionário foi autoaplicável de forma individual. Os dados foram coletados pelo pesquisador, pessoalmente, durante os meses de Novembro a Fevereiro de 2014. Para tanto, foram remetidos 230 questionários, sendo que, apenas 170 foram correspondidos, os quais compuseram nossa amostra. A mesma foi composta por médicos (65), psicólogos (17), nutricionistas (3), enfermeiros (26), técnicos de enfermagem (33), técnicos de farmácia (5), assistentes sociais (13), profissionais de Educação Física (3) e fisioterapeutas (5).

Utilizou-se um questionário autoaplicável, constituído por instrumentos validados divididos em quatro blocos:

a) Informações gerais: caracterização da idade, sexo, nível de formação, estado conjugal, peso e estatura (autorreferido), para cálculo do Índice de Massa Corporal - IMC (peso/estatura ${ }^{2}$ ). Para classificação do IMC (status de peso), utilizou-se o protocolo da Organização Mundial de Saúde6 que estabelece os pontos de corte: abaixo do peso (IMC $<18,5$ ); peso normal (IMC 18,5-24,9); sobrepeso (IMC 25,0-29,9) e obesidade (IMC > 30,0). Para fins estatísticos as categorias foram reagrupadas resultando em: peso normal: abaixo do peso e peso normal; acima do peso: sobrepeso e obesidade; b) Situação socioeconômica: por meio do instrumento adotado pela Associação Nacional de Empresa de Pesquisa - Critério de Classificação Econômica Brasil,, ${ }^{7}$ que classifica a população em estratos econômicos $A, B$, C, D e E, por meio da pontuação obtida; c) Escala de estresse no trabalho, versão resumida da "job stress scale" para o português. ${ }^{8}$ A Escala de Estresse no Trabalho possui 17 questões, divididas em três dimensões: demanda psicológica, controle (discernimento intelectual e autoridade sobre as decisões) e apoio social. Após atribuir o escore de cada questão, soma-se o total de cada dimensão, chegando-se às possibilidades de alta ou baixa demanda, alto ou baixo controle, alto ou baixo apoio social. O escore total da escala varia de 17 a 68 , sendo que, quanto mais próximo de 68 , maior risco de estresse. Realizou-se um ponto de corte em função da média obtida em todas as demandas da escala e os participantes foram classificados em dois grupos: os que ficaram acima da média como alto e abaixo da média como baixo; d) A atividade física foi avaliada por meio do questionário internacional de atividade física (IPAO - versão curta), elaborado por pesquisadores de diversos países com suporte da Organização Mundial da Saúde (OMS), como parte de um estudo multicêntrico, envolvendo 12 países. Cada país participante adaptou e validou o questionário de acordo com as características da respectiva população. ${ }^{9}$

A análise estatística foi efetuada por meio do SPSS - versão 20,0. A normalidade dos dados foi verificada pelo teste de Komogorov Smirnov. A associação das características dos participantes entre o sexo masculino e feminino foi realizada por meio do teste do Qui quadrado e a comparação da atividade física e da escala de estresse no trabalho, pelo Teste t para amostras independentes. A correlação de Pearson foi adotada para associar atividade física e estresse no trabalho. Por fim, comparou-se estresse no trabalho e nível de atividade física, utilizando-se o teste do Qui quadrado. O nível de significância estatística foi estabelecido para $p<0,05$.

\section{RESULTADOS}

As características da amostra estratificada pelo sexo, são apresentadas na tabela 1. O IMC, o nível de formação e o estrato econômico foram as variáveis que obtiveram diferença estatística significativa, sendo $(p<0,001 ; p=0,011 ; p=0,006)$, respectivamente. Foi observado que a maior parte dos participantes apresentam peso normal $(52,5 \%)$ e pertencem ao estrato econômico B $(56,9 \%)$, com destaque para as mulheres. Quanto ao nível de formação, são especialistas $(42,9 \%)$, evidenciando-se os indivíduos do sexo masculino (51,5\%). Em relação à prática de atividade física, embora não se tenha encontrado significância entre os sexos $(p=895)$, verifica-se que a maioria dos profissionais são suficientemente ativos $(58,4 \%)$.

Observa-se na tabela 2, que os homens praticam atividade física em intensidade vigorosa com maior frequência, quando comparados às mulheres $(18 \pm 44$ $\min / d)$, havendo diferença significativa $(p=0,013)$. Nas demais variáveis não foram encontradas diferenças estatísticas, porém foi verificado que os homens permanecem mais tempo sentados, nos dias de semana $(1862 \pm 783 \mathrm{~min} / \mathrm{d}$; equivalente a $\sim 6$ horas $/ \mathrm{d}) \mathrm{e}$, nos finais de semana, (749 \pm 408 ; equivalente a $\sim 6$ horas $/ d$ ), embora apresentem um tempo de caminhada e atividade física total levemente superiores $(42 \pm 41 \mathrm{~min} / \mathrm{d}$; $107 \pm 85 \mathrm{~min} / \mathrm{d}$ ), respectivamente. 
ATIVIDADE FÍSICA RELACIONADA AO ESTRESSE NO TRABALHO DOS PROFISSIONAIS DA ÁREA DA SAÚDE MENTAL

Tabela 1 - Características pessoais de acordo com o sexo dos profissionais participantes do estudo (\%). São José-SC. 2013.

\begin{tabular}{|c|c|c|c|c|}
\hline & Total & Masculino & Feminino & Valor de $p$ \\
\hline \multicolumn{5}{|l|}{ IMC* } \\
\hline Peso normal & 52,5 & 26,6 & 70,2 & $<0,001$ \\
\hline Acima do peso & 47,5 & 73,4 & 29,8 & \\
\hline \multicolumn{5}{|l|}{ Nível de formação } \\
\hline Graduado & 24,7 & 17,6 & 29,4 & \\
\hline Especialista & 42,9 & 51,5 & 37,3 & 0,011 \\
\hline Mestre & 5,3 & 4,4 & 5,9 & \\
\hline Doutor & 7,1 & 13,2 & 2,9 & \\
\hline Técnico & 20 & 13,2 & 24,5 & \\
\hline \multicolumn{5}{|l|}{ Estado Conjugal } \\
\hline Casado(a)/morando junto & 66,5 & 72,1 & 62,7 & 0,277 \\
\hline Viúvo(a) & 0,6 & 1,5 & - & \\
\hline Separado(a)/divorciado(a) & 4,1 & 4,4 & 3,9 & \\
\hline Solteiro(a) & 28,8 & 22,1 & 33,3 & \\
\hline \multicolumn{5}{|l|}{ Estrato econômico } \\
\hline A & 39,5 & 52,2 & 31,0 & 0,006 \\
\hline B & 56,9 & 47,8 & 63,0 & \\
\hline C & 3,6 & - & 6,0 & \\
\hline \multicolumn{5}{|l|}{ Nível de atividade física } \\
\hline Insuficientemente ativo & 41,6 & 41,3 & 41,8 & 0,895 \\
\hline Suficientemente ativo* & 58,4 & 58,8 & 58,2 & \\
\hline
\end{tabular}

*IMC - Índice de massa corporal; * suficientemente ativo - ativo + muito ativo. Teste do Qui quadrado $p<0,05$.

Tabela 2 - Comparação da atividade física entre os sexos dos profissionais participantes do estudo (média \pm desvio padrão). São José-SC. 2013.

\begin{tabular}{|c|c|c|c|c|}
\hline & Total & Masculino & Feminino & Valor de $p$ \\
\hline Caminhada $(\min / \mathrm{d})$ & $42 \pm 41$ & $44 \pm 41$ & $41 \pm 41$ & 0,877 \\
\hline A.F. Moderada (min/d) & $48 \pm 49$ & $44 \pm 45$ & $51 \pm 52$ & 0,828 \\
\hline A.F Vigorosa (min/d) & $14 \pm 34$ & $18 \pm 44$ & $11 \pm 27$ & 0,013 \\
\hline A.F Moderada + Vigorosa $(\min / \mathrm{d})$ & $63 \pm 66$ & $63 \pm 71$ & $63 \pm 64$ & 0,950 \\
\hline A.F Total $(\mathrm{min} / \mathrm{d})$ & $105 \pm 84$ & $107 \pm 85$ & $104 \pm 84$ & 0,882 \\
\hline Minutos sentado (semana) & $1844 \pm 717$ & $1862 \pm 783$ & $1831 \pm 672$ & 0,269 \\
\hline Minutos sentado (final de semana) & $738 \pm 380$ & $749 \pm 408$ & $731 \pm 362$ & 0,724 \\
\hline
\end{tabular}

A.F: Atividade Física; min/d: minutos/dia. Teste t para amostras independentes $-p<0,05$

Tabela 3 - Comparação do estresse no trabalho em função do sexo dos profissionais participantes do estudo (média e desvio padrão). São José-SC. 2013.

\begin{tabular}{|c|c|c|c|c|}
\hline & Total & Masculino & Feminino & Valor de $p$ \\
\hline Demanda psicológica & $10,28 \pm 2,2$ & $10,20 \pm 2,5$ & $10,34 \pm 2,1$ & 0,680 \\
\hline Controle & $10,81 \pm 2,1$ & $10,67 \pm 2,3$ & $10,91 \pm 1,7$ & 0,235 \\
\hline Apoio social & $12,45 \pm 2,6$ & $12,52 \pm 2,6$ & $12,40 \pm 2,6$ & 0,521 \\
\hline Escala total & $33,55 \pm 4,6$ & $33,41 \pm 5,6$ & $33,65 \pm 3,7$ & 0,355 \\
\hline
\end{tabular}

Teste $t$ para amostras independentes $-p<0,05$.

Tabela 4 - Comparação do estresse no trabalho em função do sexo dos profissionais participantes do estudo (média e desvio padrão). São José-SC. 2013.

\begin{tabular}{|c|c|c|c|c|}
\hline & Demanda psicológica & Controle & Apoio social & Escala total \\
\hline Caminhada $(\min / \mathrm{d})$ & $-0,126$ & $0,179 *$ & 0,138 & 0,108 \\
\hline Moderada $(\min / \mathrm{d})$ & $-0,042$ & 0,064 & 0,091 & 0,067 \\
\hline Vigorosa (min/d) & 0,076 & $-0,256 * *$ & $-0,055$ & $-0,122$ \\
\hline Moderada + vigorosa $(\mathrm{min} / \mathrm{d})$ & 0,009 & $-0,086$ & 0,039 & $-0,014$ \\
\hline AF total $(\mathrm{min} / \mathrm{d})$ & $-0,065$ & 0,025 & 0,097 & 0,038 \\
\hline
\end{tabular}


Tabela 2 - Comparação entre estresse no trabalho e nível de atividade física dos profissionais participantes do estudo (\%). São José-SC. 2013.

\begin{tabular}{|c|c|c|c|c|}
\hline & Total & Insuficientemente ativo & Suficientemente ativo & Valor de $p$ \\
\hline \multicolumn{5}{|c|}{ Demanda psicológica } \\
\hline Baixo & 57,1 & 56,7 & 57,4 & 0,926 \\
\hline Alto & 42,9 & 43,3 & 42,6 & \\
\hline \multicolumn{5}{|l|}{ Controle } \\
\hline Baixo & 37,9 & 40,3 & 36,2 & 0,595 \\
\hline Alto & 62,1 & 59,7 & 63,8 & \\
\hline \multicolumn{5}{|c|}{ Apoio social } \\
\hline Baixo & 49,1 & 53,7 & 45,7 & 0,318 \\
\hline Alto & 50,9 & 46,3 & 54,3 & \\
\hline \multicolumn{5}{|l|}{ Escala total } \\
\hline Baixo & 39,8 & 40,3 & 39,4 & 0,905 \\
\hline Alto & 60,2 & 59,7 & 60,6 & \\
\hline
\end{tabular}

A escala de estresse no trabalho entre os sexos é apresentada na tabela 3. Não foram identificadas diferenças significativas, entre os sexos, uma vez que foi identificado um baixo nível de estresse entre os participantes do estudo.

Observou-se, na tabela 4, que a demanda controle apresentou uma correlação positiva com a caminhada $(r=0,179 ; p<0,05)$ e uma correlação negativa com a atividade física vigorosa $(r=-0,256 ; p<0,01)$.

$\mathrm{Na}$ tabela 5, encontra-se a comparação entre as dimensões da escala de estresse no trabalho, classificadas como baixo e alto e o nível de atividade física, dividido em insuficientemente ativo e suficientemente ativo. Não houve diferença estatística entre as variáveis. Entretanto, a maior parte dos indivíduos apresentam baixo controle, baixo apoio social e baixa demanda psicológica são suficientemente ativos $(57,4 \% ; 62,1 \% ; 50,9 \%)$, respectivamente.

\section{DISCUSSÃO}

O principal objetivo do estudo foi investigar a relação entre atividade física e estresse no trabalho dos profissionais que atendem pacientes com transtornos mentais e dependentes químicos de São José. O ambiente de trabalho na área da saúde mental, como se constatou, pode desenvolver algumas doenças, entre elas o estresse, devido às atividades desenvolvidas exigirem alto grau de responsabilidade e qualificação, com desgaste emocional intenso. Neste estudo, os profissionais apresentam um baixo nível de estresse, caracterizando-se por ser uma população não estressada, no presente momento.

Nesta perspectiva, os resultados do estudo demonstraram que o estresse no trabalho mostrou-se semelhante entre os homens e mulheres. O nível socioeconômico, pode ser um importante fator em relação a semelhança do estresse entre homens e mulheres, visto que a maioria dos participantes deste estudo pertencem ao estrato econômico $B$, o que possibilita maior dedicação dos profissionais à atividades de lazer, podendo contribuir para menores níveis de estresse e maior satisfação no trabalho. ${ }^{10}$

O estresse, quando presente, pode desencadear uma série de doenças. Se nada é feito para aliviar a tensão, o indivíduo pode se sentir exaurido, sem energia e com estado de espirito depressivo. ${ }^{11}$ É importante ressaltar que a prática de atividade física contribui para o controle ou até mesmo para minimizar o estresse e seus efeitos negativos à saúde, atuando no aumento da autoestima e autoeficácia, fatores que ajudam a reduzir os níveis de tensão. ${ }^{12}$

Em nosso estudo, foi constatado que a maioria dos profissionais são suficientemente ativos. Comparando-se o nível de atividade física entre os sexos, verificou-se que o sexo masculino, por sua vez, mostrou-se mais ativo, com destaque para a prática de atividade física em intensidade vigorosa. Em contrapartida, Brito et al., ${ }^{13}$ em um estudo com professores, verificaram que, no sexo masculino, o nível de atividade física foi menor em relação ao feminino. Entretanto, neste estudo foi observado que os homens passam mais tempo sentados nos dias de semana e nos finais de semana em comparação as mulheres, mostrando que, apesar de serem ativos, apresentam um comportamento sedentário.

A atividade física tem relação direta com a diminuição dos níveis de estresse. É considerada como um poderoso estímulo para a liberação de fatores vaso relaxantes produzidos pelo endotélio muscular, acarretando também na redução da pressão arterial. ${ }^{14}$ Além disso, é uma ferramenta eficiente para otimizar os processos relacionados com a redução dos fatores de risco inerentes à inatividade, representando assim um processo social e político abrangente, que não abrange ações voltadas ao fortalecimento das habilidades e capacidades dos indivíduos, mas também ações dirigidas à mudança social, ambiental e econômico, a fim de aliviar o seu impacto na saúde..$^{15}$

Quanto à intensidade da atividade física praticada, foi identificado resultado significativo em intensidade vigorosa, com maiores médias, para o sexo masculino. É observado que homens, que praticam atividade física vigorosa, com gasto calórico superior a 3,500kcal/ semana demonstram mudança no estilo de vida, apresentando risco relativo de morte reduzido para $41 \% .{ }^{16}$

É válido ressaltar que os profissionais deste estudo, em geral, realizam mais que o recomendado para a população adulta, ${ }^{17}$ sendo que a atividade física moderada+vigorosa é a mais praticada entre todas as modalidades. Tal fato pode ser elucidado, pois todos os participantes possuem uma alta escolaridade, grande parte com título de especialista e um estrato econômico elevado, a maioria entre a classe A e B. Segundo Camões e Lopes, ${ }^{18}$ indivíduos com maior grau de escolaridade e nível socioeconômico, apresentam, 
na maioria das vezes, níveis mais elevados de atividade física, devido à oportunidade de acesso à instruções de saúde. Ademais, por se tratar de profissionais da saúde e lidarem diretamente com esta área, sabem da importância da prática da atividade física e seus benefícios, não só físico, mas também mental e emocional.

Ao relacionar nível de atividade física e estresse no trabalho, não foram observadas diferenças significativas entre as variáveis. Embora a atividade física não tenha exercido relação direta sobre o estresse neste estudo, talvez possa ter auxiliado na vida dos profissionais, agindo de forma menos danosa à saúde. Todavia, observa-se para uma relação inversamente proporcional entre a atividade física e o estresse..$^{19}$

Ressalta-se que, no presente estudo, houve uma correlação positiva entre a demanda controle e caminhada, justificando a importância da atividade física em relação ao trabalho, amenizando a sobrecarga imposta ao profissional da área da saúde. É importante esclarecer que a demanda controle está relacionada ao respeito, ao uso e desenvolvimento de habilidades e à autoridade para tomada de decisão, sobre o processo de trabalho. ${ }^{8}$

Pode-se perceber no presente estudo que, de uma forma geral, os profissionais possuem nível de estresse baixo. A atividade física realizada parece não ser suficiente para o controle do estresse neste momento, o que sugere a influência negativa de outras variáveis como o número de filhos, a percepção de saúde, o uso de medicamentos, o lazer, e o tempo de trabalho na instituição, que não foram objeto deste estudo.

O presente estudo foi avaliado por meio de um questionário, que representa uma limitação deste estudo, uma vez que este método de avaliação está associado com uma estimativa de erro de mais de (35-50\%). ${ }^{20}$ Recomendações para a atividade de melhoria da saúde física são, no entanto, com base em estudos epidemiológicos, os que utilizaram a mesma metodologia, ou seja, com um erro semelhante.

\section{CONSIDERACִÕES FINAIS}

Durante a construção deste estudo, foi identificado baixo nível de estresse nos profissionais que atendem pacientes com transtornos mentais e dependentes químicos, evidenciado pela baixa demanda psicológica, não verificando que, tanto os homens, quanto as mulheres são acometidos pelo estresse de forma semelhante.

Finalmente, no presente estudo foi observada uma relação negativa (inversa) entre atividade vigorosa e demanda de controle. Porém, nas demais variáveis do estresse não verificou-se essa relação, inclusive entre os demais componentes da atividade física. Portanto, a atividade física não está relacionada ao estresse neste estudo.

\section{REFERÊNCIAS}

1. Camelo SHH, Angerami ELS. Riscos Psicossociais no trabalho que podem levar ao estresse:uma análise da literatura. Ciênc Cuid Saúde 2008; 7(2): 232-240. DOI: http://dx.doi. org/10.4025/cienccuidsaude.v7i2.5010.

2. Ebling $M$, Carlotto SM. Síndrome de Burnout e fatores associados entre profissionais de saúde de um hospital público. Trends Pychiatry Psychother 2010; 34(2): 93-100. DOI: http://dx.doi.org/10.1590/S2237-60892012000200008.
3. Hanzelmann RS, Passos JP. Imagens de Enfermagem e representações sobre o estresse e influência sobre a atividade de trabalho. Rev Esc Enferm USP 2010; 44(3): 694-701. DOI: http://dx.doi.org/10.1590/S0080-62342010000300020.

4. Silva AA, Souza JMP, Borges FNS, Fischer FM. Qualidade de vida relacionada à saúde e condições de trabalho entre os provedores de enfermagem. Rev Saúde Pública 2010; 44(4): 718-725. DOI: http://dx.doi.org/10.1590/S003489102010000400016.

5. Jodas DA, Haddad MCL. Síndrome do Burnout em trabalhadores de enfermagem de um pronto socorro de hospital universitário. Acta Paul Enferm 2009; 22(2): 192-197. DOI: http://dx.doi.org/10.1590/S0103-21002009000200012.

6. WHO, World Health Organization. The International Classification of adult underweight, overweight and obesity according to BMI, 2004. Disponível em: http://apps.who.int/bmi/index. jsp?introPage=intro_3.html. Acesso em: 23 de julho 2013.

7. ABEP. Critério de Classificação Econômica Brasil. Associação Brasileira de Empresas de Pesquisa, 2010.

8. Alves MGM, Chor D, Faerstein E, Lopes CS, Werneck GL. Versão resumida da "job stress scale": adaptação para o português. Rev Saúde Pública 2004; 38(2): 164-171. DOI: http://dx.doi.org/10.1590/S0034-89102004000200003.

9. Pardini R, Matsudo SMM, Matsudo VKR, Araujo T, Andrade E, Braggion G. Validation of international physical activity questionnaire (IPAQ): pilot study in brazilian young adults. Med Sci Sports Exerc 1997; 29(6): S5-S9.

10. Souza WC, Silva AMM. A influência de fatores de personalidade e de organização do trabalho no burnout em profissionais da saúde. Estud Psicol 2002; 19(1): 37-38. DOI: http://dx.doi.org/10.1590/S0103-166X2002000100004.

11. Passos JC, Silva EL, Carvalho MMC. Estresse no centro cirúrgico: uma realidade dos profissionais de enfermagem. Rev Pesq Saúde 2010; 11(2): 35-38.

12. Truccolo AB, Maduro PA, Feijó EA. Fatores motivacionais de adesão a grupos de corrida. Motriz 2008; 14(2): 108-114.

13. Brito WF, Santos CL, Marcolongo AA, Campos MD, Bocalini DS, Antonio EL, Silva Junior JA, Tucci PJ, Serra AJ. Physical activity levels in public school teachers. Rev Saúde Pública 2012; 46(1): 104-109. DOI: http://dx.doi.org/10.1590/ S0034-89102012000100013.

14. Zago AS, Zanesco A. Óxido nítrico, doenças cardiovasculares e exercício físico. Arq Bras Cardiol 2006; 87(6): 264-270. DOI: http://dx.doi.org/10.1590/S0066-782X2006001900029.

15. Claros JAV, Álvarez CV, Cuellar CS, Mora MLA. Actividad física: estrategia de promoción de la salud. Hacia la Promoción de la Salud 2011; 16(1): 202-218.

16. Pitanga FJG. Epidemiologia, atividade física e saúde. Rev Bras Ciên e Mov 2002; 10(3): 49-54.

17. Who. World health organization. Global recommendations on physical activity for health, 18-64 years old. 2011. Disponível em: http://www.who.int/dietphysicalactivity/physical-activityrecommendations-18-64years.pdf. Acesso em: 15 jun. 2011.

18. Camões $M$, Lopes $C$. Fatores associados à atividade física na população portuguesa. Rev Saúde Pública 2008; 42(2): 208-216. DOI: http://dx.doi.org/10.1590/S003489102008000200004.

19. Canova KJ, Porto JB. O impacto dos valores organizacionais no estresse ocupacional: um estudo com professores do ensino médio. Rev Adm Mackenzie 2010; 11(5): 4-31. DOI: http://dx.doi.org/10.1590/S1678-69712010000500002.

20. Welk G. Physical Activity Assessments for Health-Related Research. Champaign: Human Kinetics, 2002. 